\title{
LINEAGEOS ANDROID OPEN SOURCE MOBILE OPERATING SYSTEM : STRENGTHS AND CHALLENGES
}

\author{
by
}

Rajasekhar Ponakala

A special study report submitted in partial fulfillment of the requirements for the degree of Master of Engineering in Information and Communication Technology

$\begin{array}{ll}\text { Examination Committee: } & \text { Dr. Matthew N. Dailey (Chairperson) } \\ & \text { Dr. Mongkol Ekpanyapong (Member) } \\ & \text { Dr. Attaphongse Taparugssanagorn (Member) }\end{array}$

Nationality: Indian

Previous Degree: Bachelor of Technology in Electronics and Communication Engineering Jawaharlal Nehru Technological University Hyderabad, India

Scholarship Donor: AIT Fellowship

Asian Institute of Technology

School of Engineering and Technology

Thailand

December 2017 


\section{Acknowledgments}

I would like to thank my advisor Dr. Matthew N. Dailey for the valuable suggestions, guidance, and encouragement that helped me to complete my special study.

I would also like to thank the committee members, Dr. Attaphongse Taparugssanagorn and Dr. Mongkol Ekpanyapong, for their valuable comments and suggestions for this report. I would also like to thank my friends in the ICT department at AIT for their support.

Last but not the least, I would also like to thank my parents, for their love, support, and encouragement.

Rajasekhar Ponakala

December 2017 


\begin{abstract}
The Android Open Source Project (AOSP) is a community-driven model for the development of mobile operating system platforms. Developers around the world distribute and share code, enabling others to use it and develop their own AOSP variants. This special study is aimed at identifying opportunities and challenges in developing a completely free and open source mobile operating system based on AOSP.

LineageOS, an active fork of the now-defunct CyanogenMod AOSP variant, has been ported to several different devices since its initial release. LineageOS ships with a complete set of binary firmware and shows the way for developers around the world to develop alternative platforms to Google Mobile Services.

In this special study, I describe the installation of LineageOS on a OnePlus One mobile device. I first describe the installation of a pre-built LineageOS image to the device, and then I explain how i compiled the available source to build my own LineageOS image for the device. Source compilation provides a more complete understanding of device-level OS builds.

I also discuss various alternatives to Google Mobile Services. All the available alternative services are in the development stage. A promising approach is the reverse engineering microG project, which is a free and open source re-implementation of Google Mobile Services. Such alternatives to Google's services, meaning client-side free and open source applications and libraries, provide an excellent base upon which can build completely free and open source background services as well as applications on mobile devices.
\end{abstract}




\section{Table of Contents}

\section{Chapter Title}

\section{Page}

Title Page

Acknowledgments

Abstract

Table of Contents

List of Figures

List of Tables

Introduction

1.1 Background

1.2 Problem Statement

1.3 Objectives

1.4 Organisation of the report

Literature Review 4

2.1 Mobile Operating Systems 4

2.2 Google Mobile Services and other alternative applications 4

2.3 Application Repositories 6

2.4 microG Project 7

2.5 Related works on Android Open Source Project 7

3 Methodology 9

3.1 Device Partitions 9

3.2 Custom Roms 9

3.3 Android SDK Platform Tools $\quad 10$

3.4 Boot-loader and Recovery Mode 10

$\begin{array}{ll}3.5 \text { Wiping Partitions } & 10\end{array}$

3.6 Flashing LineageOS 11

3.7 Open GApps 11

$\begin{array}{ll}3.8 \text { Installing microG suite } & 12\end{array}$

$4 \quad$ Results 13

4.1 Pre-build Installation $\quad 13$

4.2 Source Compilation 13

5 Discussion and Conclusion $\quad 21$

$\begin{array}{lll}5.1 \text { Discussion } & 21\end{array}$

5.2 Conclusion 21

6 References 22 


\section{List of Figures}

$\begin{array}{lll}\text { Figure Title Page } & \text { Pag }\end{array}$

$2.1 \quad$ Overview of Play Services 5

4.1 Main build tree 14

4.2 Final build files 15

$\begin{array}{lll}4.3 & \text { Proprietary files }-1 & 18\end{array}$

4.4 Proprietary files (listing 2) 19 


\section{List of Tables}

Table Title

Page

$2.1 \quad$ Work plan for research 8

4.1 OnePlus One mobile specifications 20 


\section{Chapter 1}

\section{Introduction}

This chapter provides information about the Background, Problem Statement and Objective of the study.

\subsection{Background}

The Android Operating System (OS) is a free and open source, fully customizable mobile OS platform. The Android Open Source Project (AOSP), which provides a framework for the development of Android, uses a community-driven model for the improvement of mobile operating system platforms. Developers around the world can distribute and share code. This helps others to use the shared code and develop their own AOSP variants. In November 2007, AOSP was announced. Since the initial release of the AOSP, many popular variants have been introduced. AOSP is led by Google and the Open Handset Alliance (OHA). Android source code is released under an open source license, but paradoxically, most Android devices ship with a combination of free and open source and proprietary firmware, especially the firmware required for accessing Google Play Services.

Google Play Services, generally referred to as Google Mobile Services (GMS), has system-level control over the mobile device. It is part of the Google Apps package and is proprietary. Developers having the intention to develop an open source application and publish it on the Play store need to agree upon Google's terms, which state that a few parts of the code are proprietary. This paradoxical situation of the impossibility of launching a complete free and open source applicaton via the Play Store raises many privacy and security-related concerns.

AOSP has functioned as the baseline for all original equipment manufacturers (OEMs) and communityrun packaged firmware such as CyanogenMod. Some of the apps inside AOSP have been abandoned and then released on Google Play with a closed-source model. Many apps only function when Google Play Services are available. Examples include Gmail, YouTube, and Google Maps.

There are many distributions of Android. CyanogenMod (CM) was a popular fork of AOSP developed by Cyanogen (Cyngn) Inc. that emphasized allowing its users to modify everything on the platform. In December 2016, Cyanogen Inc. announced the discontinuation of the CyanogenMod project. OnePlus' first phone, Oneplus One, was a popular smart phone featuring Cyanogen OS when it shipped in 2015. Builds builds are still available. Immediately after the discontinuation of CyanogenMod, LineageOS took up the effort to continue towards the goal. It is a fork of the now-defunct CyanogenMod version of Android. Similar to CM, the LineageOS project is being extended by many device-specific maintainers.

LineageOS emphasizes the delivery of a complete free and open source project. This is in contrast with the usual way to run Android hardware, in which source code is adapted by original equipment manufacturers (OEMs) that produce parts and equipment that were originally manufactured by another company. 


\subsection{Problem Statement}

This section states various problems with the Android Open Source Project in practice.

\subsubsection{Google Play Services API}

Most Android application developers rely on the Application Programming Interfaces (APIs) of Google Play services. These services include the location API, which is needed for all map-related work on a mobile device. Google Play Services is a proprietary code base; hence, nobody knows precisely how applications using it work. There are many similar APIs for which we do not have access to the full source code.

\subsubsection{Device Drivers}

For years, Google has been adding functionality not only to the AOSP code base, but also to its own proprietary binary libraries and very specific GMS (Google Mobile Services). Manufacturers must pass a compatibility test to receive official GMS support from Google. Usually, the code being tested includes device drivers that are proprietary, meaning there is no hope of looking at the code to understand what happens inside. As a user of proprietary software is not allowed to control its execution (the vendor and other third parties control it), he or she cannot prevent tracking and selling his or her information to advertising companies, and he or she is prevented from doing all possible things on the device. At a high level, users are controlled and directed to do what vendors want them to do. On a practical level, users are continually told that their devices are obsolete just because vendors fail to upgrade the firmware. Specifically, even though AOSP is free and open source software in theory, in practice most devices uses dozens of proprietary blobs to control the actual hardware.

\subsubsection{Other cases}

Most Android-based devices are manufactured with a chip-set named Qualcomm Snapdragon. This is a system on chip (SOC) semiconductor device designed and marketed by Qualcomm for mobile devices. Qualcomm develops a large number of propreitory device drivers for the SOC. Other device manufacturers have to buy the SOC and drivers from Qualcomm. For example, the H.264 video and audio codec library for the Snapdragon is actually developed by Qualcomm, who owns a patent on it. Being closed source, any flaws of the hardware-level implementation of the mobile phone are nearly impossible to fix.

Documents released by Edward Snowden have revealed that the National Security Agency (NSA) and Global Surveillance joint programs, such the program named PRISM, aims at collecting all possible information about people around the world by partnering with companies such as Microsoft, Facebook, Apple, and Google (MacAskill, 2013). LineageOS is aimed at community development that reaches the masses and provides a purely free and open source operating system. GMS is not 
provided by default in LineageOS. Instead, users can decide themselves whether or not to load it. Alternatives to GMS are in the development phase. The LineageOS developer community is focused on better free and open source operating system software for users to have a complete functional OS in their hands.

\subsection{Objectives}

The objective of this study is to understand the implications of the AOSP on technology freedom, identifying weaknesses and opportunities for LineageOS, and to experiment with LineageOS on a real device.

To achieve these objectives, I have performed following steps:

- Write a literature survey to provide a good grasp of the background.

- Perform a technology review to know more about the AOSP and identify methods to continue LineageOS on different platforms.

- Install LineageOS on a device to check device-level performance.

- Identify gaps compared to other AOSP projects.

- Make a plan for possible contribution to LineageOS to help improve the AOSP.

\subsection{Organisation of the report}

Chapter 1 provides the background, problem statement, and objectives of the study. Chapter 2 provides a literature survey. Chapter 3 describes the methodology of my study. Chapter 4 discusses preliminary results. Discussion and conclusion are in Chapter 5. 


\section{Chapter 2}

\section{Literature Review}

This chapter reviews mobile operating systems and Google Mobile Services (GMS) and compares alternatives to GMS such as independent email applications, alternative search engines, offline maps, and alternative application repositories. I also provide information regarding the previous work.

\subsection{Mobile Operating Systems}

In general, a mobile OS typically starts up when a device powers on, presenting a screen with icons or tiles that present information and provide application access. Mobile operating systems manage cellular and wireless network connectivity, as well as phone access. All of these tasks must be handled by an embedded system.

Around 1994, IBM launched the first smartphone, named IBM Simon, which only had a touchscreen, email, and PDA features. Slightly later, Symbian Ltd introduced Symbian OS, which was used by Nokia and then became the first modern mobile OS on a smartphone with the launch of the Ericsson R380 mobile phone. Around 2008, the Open Handset Alliance (OHA) released Android 1.0 based on the Linux kernel on the HTC Dream (T-Mobile G1) as the first Android phone. Android's first version had features including the GMS from the beginning.

AOSP, being an open source developer community spread around the world, enables introduction of different custom ROMs enabling users to modify the system. LineageOS, which is a recent fork of the defunct of CyanogenMod ROM, allows users to side-load GMS or leave it out.

\subsection{Google Mobile Services and other alternative applications}

Google Mobile Services (GMS) includes applications and services provided by Google that enhance the user's mobile experience. Applications such as Gmail, Chrome, Google Maps, YouTube, Google Docs, and the Play Store are pre-installed in stock Android versions. These are closed source applications. There are many privacy and data mining issues with GMS because they ships many proprietary blobs with unknown functions in the software and device drivers. Google Play Services has two major components: client library and external Android Package Kit (APK) files. The client library contains interfaces to the individual Google services and allows them to obtain authorization from users to gain access to Google Play Services with their credentials. It also contains APIs that allows resolving any issues at runtime, such as a missing, disabled, or out-of-date Google Play services APKs. The Google Play Services APK contains the individual Google services and runs as a background service in the OS. Figure 2.1 shows an overview of the Google Play Services and their operation. 


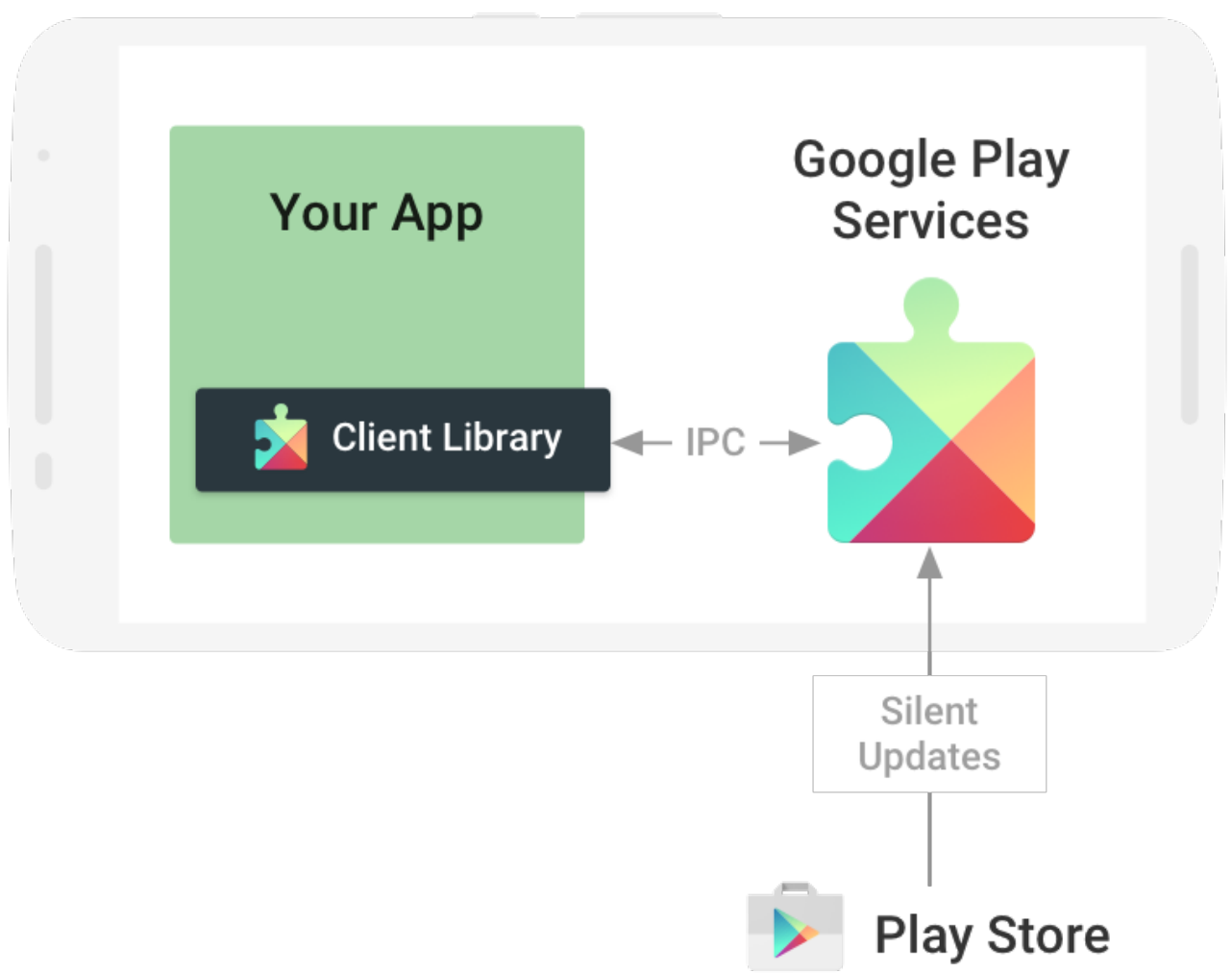

Figure 2.1: Overview of Play Services

(https://developers.google.com/android/guides/overview)

Independent Email Applications such as K-9 Mail, Tutanota, and ProtanMail help developers to review and modify their source as intended under a free and open source model. K-9 Mail is the most popular alternative mail service has several features. Tutanota and Proton are also being the popular alternative mail services has the end-to-end encryption infrastructure. As GMS is unable to assuage user's privacy concerns, these kind of alternative email applications are necessary.

There are different search engines available as alternatives to Google search. All of these search engines are based on search engine optimization (SEO) techniques. DuckDuckGo (DDG) is a popular open source alternative to Google search. It emphasizes protecting searchers' privacy and avoiding personalized search results. DDG distinguishes itself from other search engines by claiming that it does not profile its users and deliberately shows all the users same search results for a given search term. It emphasizes returning the best results rather than the most results and generates those results from over 400 individual sources. It includes key crowdsourced sites such as Wikipedia and other search engines such as Bing, Yahoo!, Yandex, and Yummly.

DDG has an interesting feature, "!Bang" syntax, which allows searchers to use keywords from a menu list and directly search over different available sites. There is also the possibility of writing DDG tools on the "DDG hack" platform. Some parts of DDG's source code is free software is hosted at GitHub under the Apache 2.0 License, but the core is proprietary. It is used by a few corporations 
as a default option in the provided list. On 18 September 2014, Apple included DDG in its Safari browser as an optional search engine (Schwartz, 2014). On 10 November 2014, Mozilla added DDG as a search option to Firefox 33.1 (Mozilla, 2014). On 30 May 2016, The Tor Project made DDG the default search engine for Tor Browser 6.0 (Johnson, 2016). DDG earns revenue by providing ads from the Yahoo-bing search alliance and also through partner relationship with Amazon and ebay.

For alternative Maps utilities, open street mapping is the popular model. The open street mapping (OSM) community has developed capabilities for offline maps. This had led to a great deal of growth in the community. OSM is a collaborative project to create a free editable map of world. The creation and growth of OSM has been motivated by restrictions on use or availability of map information across the world, and the advent of inexpensive portable satellite navigation devices. OSM is considered a prominent example of volunteered geographic information (Anderson, 2006).

Applications such as Maps.Me and OsmAnd provide offline maps and free navigation utilities. They has been developed in the free and open source model. Maps.Me features includes, offline map data, GPS support, offline search, offline routes for cars and by foot, a Map Editor, Bookmark, Locations, bookmarks sharing, and KML import. The OsmAnd features includ, navigation, map viewing, using OSM and Wikipedia data, safety features, bicycle and pedestrian features, and directly contributing to OSM.

\subsection{Application Repositories}

Most of the apps in the Google Play Store are not open source, and many of them are loaded with proprietary blobs and many try to gather information about their users. There are a few independent app stores that allow open source software. One such implementation is F-Droid. F-Droid is a software repository (or app store) for Android applications, similar to the Google Play store. The main repository hosted by the project, contains apps that are free and open-source software. Applications can be browsed and installed from the F-Droid website or client app. There is no need to register for an account. Applications incorporating advertising, user tracking, or dependence on non-free software must inform users in the app descriptions. The website offers the source code of the applications it hosts, and the software running the F-Droid server is also open, allowing anyone to set up their own app repository. This software repository is licensed under GNU GPLv3+ (Nardi, 2012). 


\section{4 microG Project}

The microG project is a free and open source re-implementation of Googles proprietary Android client side apps and libraries. Different free and open source applications require Googles proprietary libraries to be installed. To solve this problems, different scenarios led to the development of a free and open source software clone of Googles proprietary core libraries and applications. The main idea of this project was to build a free and open source replacement for all proprietary parts injected by Google into the Android ecosystem. The components of this project are shown below.

- Service Core (GmsCore): This is a library application that provides functionality to run Google Play Services or the Google Maps Android API.

- Services Framework Proxy (GsfProxy): This is a utility that allow apps developed for Google Cloud to Device Messaging (C2DM) to use the compatible Google Cloud Messaging service included with GmsCore.

- Unified Network Location Provider (UnifiedNlp): This is a library that provides Wi-Fi and cell-tower-based geolocation to applications that use Googles network location provider. It is included in GmsCore but can also run independently on most Android systems.

- Maps API (mapsv1): This is a system library which provides the same functionality as the now deprecated Google Maps API v1.

- Store (Phonesky): This is a front-end application providing access to the Google Play Store to download and update applications.

\subsection{Related works on Android Open Source Project}

There have been many projects building on the AOSP demonstrating tasks such as reverse engineering, exploring the Play Store, and privacy-and security-related aspects of the project. Table 2.4 shows this related works. 
Table 2.1: Work plan for research

\begin{tabular}{|c|c|c|}
\hline SL & Reference & Paper Discussion \\
\hline 1 & (Viennot, Garcia, \& Nieh, 2014) & $\begin{array}{l}\text { Developed a method for crawling the Play } \\
\text { Store applications and observing 3rd party } \\
\text { application usage from users. The author } \\
\text { also provide interesting methods for reverse } \\
\text { engineering the Play Store. }\end{array}$ \\
\hline 2 & (Erturk, 2013) & $\begin{array}{l}\text { Discusses security and privacy related } \\
\text { issues regrading Android adware. Also } \\
\text { proposes developing an open source mobile } \\
\text { application platform to reduce the security } \\
\text { vulnerabilities. }\end{array}$ \\
\hline 3 & (Khokhlov \& Reznik, 2017) & $\begin{array}{l}\text { Discusses data security evaluation for } \\
\text { Android mobile devices. Provides useful } \\
\text { information about sensor data manipulation } \\
\text { determined through different evaluation } \\
\text { metrics. }\end{array}$ \\
\hline
\end{tabular}




\section{Chapter 3}

\section{Methodology}

This chapter provides information about the implementation of LineageOS on the Oneplus One device. It mainly focuses on Device Partitioning, Custom ROMs, Android SDK Platform Tools, Bootloader and Recovery Mode, Wiping Partitions, Flashing LineageOS, and Open GApps.

\subsection{Device Partitions}

All Android devices follow a standard internal storage partition tree. As Oneplus One is an Androidbased mobile device, it follows the same kind of partitioning. A partition tree usually consists of partitions such as boot, system, recovery, data, cache, and misc. In addition to these partitions, the device also has a sdcard partition. Following are some individual details about the above-mentioned partitions.

- boot: This partition is essential for a device to boot. It comprises of the kernel and the ramdisk.

- system: This partition stores the entire operating system, other than the kernel and the ramdisk. This includes the Android user interface in addition to all the applications that are pre-installed on the device.

- recovery: This is an alternative boot partition that lets users boot the device into a recovery console for advanced recovery and maintenance operations.

- data: This partition contains the user's data. All the contacts, messages, settings, and apps are installed by default on this partition.

- cache: This partition stores frequently-accessed data and app components.

- misc: This partition stores miscellaneous system settings. This includes, settings such as carrier or region ID (CID), USB configuration, and a few hardware settings. This partition changes from device to device.

- sdcard: Usually, this is not a partition on the internal memory storage device but a mounted seperate SD card partition storing media, documents, and Custom ROMs.

\subsection{Custom Roms}

LineageOS, which continues the defunct CyanogenMod project, is a custom ROM. It gives different facilities to users to modify the system according to their interests. Currently, LineageOS has custom builds for 29 devices, each of which has different versions. LineageOS developers also provides a procedure to build LineageOS for specific user devices. 
At present, OnePlus company has five different devices, and OnePlus One is the first version of their flagship product line. For each of these devices, LineageOS has different nightly builds, which are maintained regularly. OnePlus One's nightly builds are named under the codename "bacon". In order to proceed with the installation, users should download a nightly build.

At first, OnePlus One was pre-installed with CyanogenMod OS. By December 2016, there were no future stock updates from the company. During that period, the device had CyanogenMod 13.1 as the stock OS. Before installing LineageOS, it is recommended to flash the unofficial CyanogenMod 13.1.2 OS first, as it upgrades to the latest firmware. Before installing any custom ROM, it is suggested to have the latest firmware on the device. Android SDK platform tools help to interact with the device shell and further interact with wiping partitions, flashing the OS, and other tasks.

\subsection{Android SDK Platform Tools}

Android SDK Platform Tools are basic to developing Android applications. The toolset include tools that interface with the Android platform, such as adb, fastboot, and systrace. These tools help unlock the device boot-loader and allow flashing it with a new system image. Using fastboot, a device can be flashed with a recovery file, then adb can be used to flash a custom ROM through the recovery mode. adb also allows the device shell to be accessed.

\subsection{Boot-loader and Recovery Mode}

In general, the bootloader is a software program that activates an operating system. We can utilize the fastboot tool to switch to bootloader mode in order to flash a new version of the OS. This involves a complete format of the partition tree. To perform privileged shell-level activities, we must unlock the bootloader in order to give root access to the system partitions.

As mentioned earlier, the recovery partition is a separate partition on the system. It helps users to recover a damaged system with the help of tools. Besides the stock recovery mode, a device can be loaded with a custom recovery tool. Usually, custom recovery provides more functions than the stock recovery. There are many custom recoveries, among which ClockworkMod (CWM) and TeamWin (TWRP) are the most popular. CWM is a software company, whereas TWRP is an open source community project.

\subsection{Wiping Partitions}

In order to flash a custom ROM, the device's partitions should first be formatted. Partitions such as data, system, and cache must be wiped from the system. This is because most data is stored in those partitions. In many of the cases, normal wiping works, but if partitions are not wiped correctly, a hard wipe may be required; this means a full format of the device. OnePlus One uses the ext 4 format for 
the partition tree, and it requires a hard wipe to format the partition tree, because this requires several back and forth operations regarding the OS.

\subsection{Flashing LineageOS}

Flashing LineageOS on OnePlus One requires a few basic steps. As mentioned earlier, we should first upgrade to the stock version of CM 13.1.2 and follow by flashing an experimental migration build of LineageOS. This is because of the firmware included in the last stock version. LineageOS builds prior to that stock version are deprecated, and now version 14 nightly builds should be used for all devices.

We must make sure to have a full backup of the system, as data is not recovered after formatting the storage device. Also, the device needs a full charge before proceeding with the installation.

The XDA-developer community maintains the unofficial stock CM 13.1.2 version in the form of downloadable zip file. We must also make sure to download the Android SDK platform tools for Debian GNU/Linux (now it is only implemented in this environment). We must place the downloaded CM 13.1.2 zip in the SDK tools directory.

We enter fastboot mode and flash the zip file before connecting it to the laptop. TWRP is suggested on user forums. We must check the device information to make sure the flashed OS is as expected. Next, to flash LineageOS, we must make sure to download the experimental migration build from CM. Finally, by repeating the above steps, LineageOS can be successfully installed. Hereafter, nightly builds can be received as updates occur.

\subsection{Open GApps}

It is possible to install the bundled Google Apps package. This package depends on Google Mobile Services. LineageOS offers users a choice to side-load these bundled GApps. 


\subsection{Installing microG suite}

To enable the services offered by the microG project, we have to install the microG-LineageOS build of the OS. To flash this version of LineageOS, we follow same steps previously described earlier to flash LineageOS. Utilizing the Android platforms tools, we can install the zip file from the TWRP recovery mode.

One issue in using microG is that we have to enable signature spoofing in the system application settings. Signature spoofing allows applications to behave as if they have been signed by a third party. This means that whenever one application asks the operating system for the certificate used to sign an installed package and that package uses signature spoofing, instead of the original certificate associated with the app, a spoofed certificate is returned. This certificate has to be announced in the AndroidManifest file, and the app is required to request the android-permission-FAKE-SIGNATURE file permission. These signatures are used to verify the integrity of a package and to ensure that the new version is from the same author when the system updates the app. The verification process normally relies on the fact that, only one author has access to the private key used to sign an app. A different author is not able to sign an app using the same key, because he does not have access to it, and thus cannot provide update to another authors application. Signatures are thus an essential part of the Android package management security system.

Besides the official Play store app, there are other application stores that providing utilities similar to the Play Store, and the Yalp store is one of them. Yalp Store lets us download apps from the Google Play Store as apk files. It can be installed as a system app to gain background package installation permission. It is independent from the Google Services framework. It uses the same remote API that the Play store app uses. 


\section{Chapter 4}

\section{Results}

This chapter gives results for the work that was done in chapter 3. It has the two method results which are based on the pre-build installation and source code compilation.

\subsection{Pre-build Installation}

First, I attempted installation through the pre-build images from the official LineageOS website. This process utilizes the Android studio platform tools. As the process involves two upgrades, first the Oneplus One needs to be upgraded to the LineageOS experimental build, then we follow with the nightly update as they occur. The whole process took two hours on a $2.5 \mathrm{GHz}$ CPU with 3GB RAM. Table 4.1 shows the specifications of the OnePlus One mobile phone.

\subsection{Source Compilation}

Next I fetched the source from the official LineageOS project page in Github. I did the build on a virtualbox with Ubuntu 16.04 as the host system. The process took nine hours to build the entire image on a $3.10 \mathrm{GHz}$ CPU-i5-4440 with RAM of 5GB. In order to build proprietary modules, I extracted those modules sources from existing LineageOS device. Here, I used the pre-build image for the OnePlus One device to extract the sources of the proprietary modules. The code is available in official LineageOS Github page. The Figures 4.1-4.4 show the post execution directories of the LineageOS source compilation. 


\subsubsection{Main tree of build files}

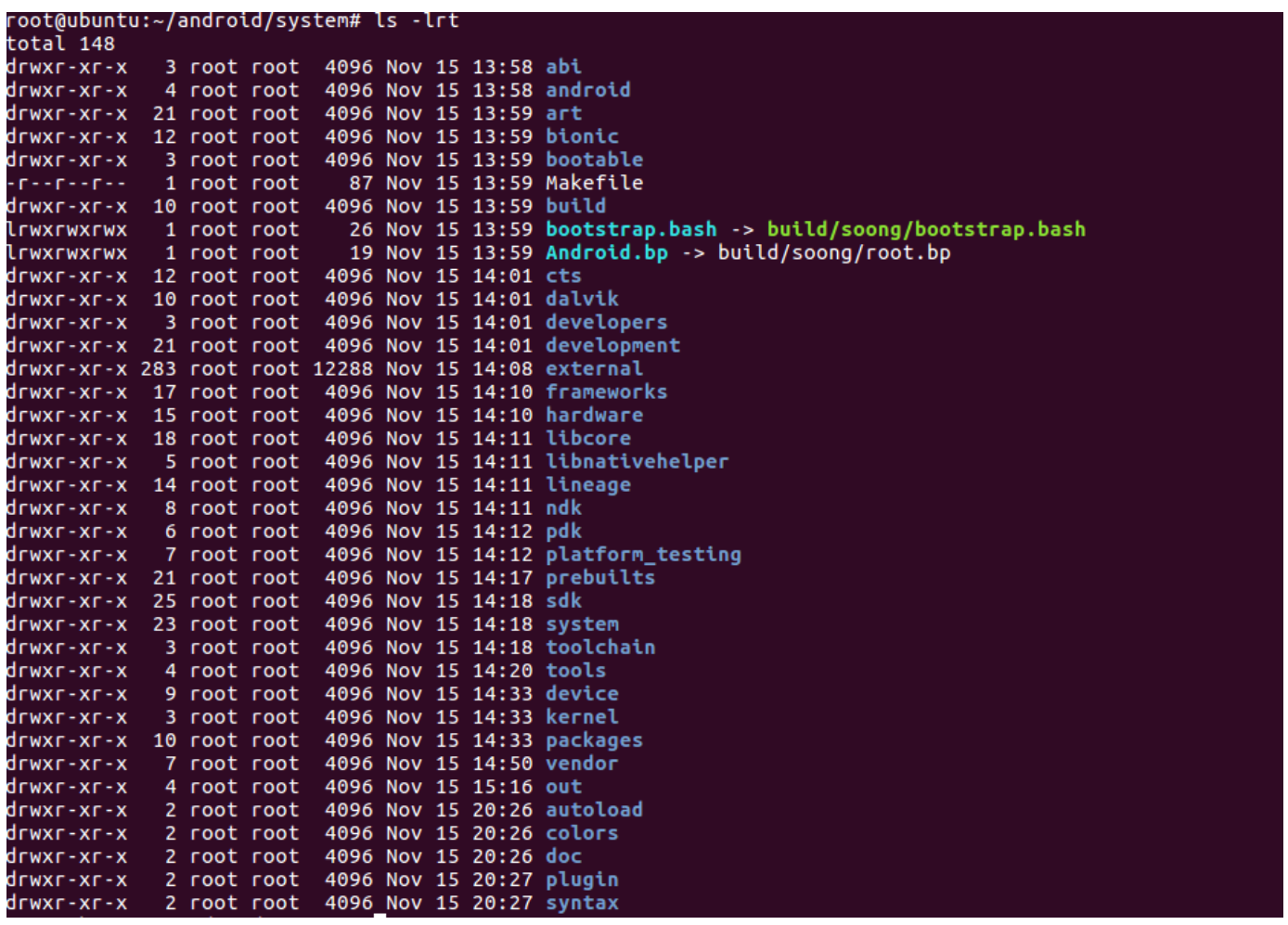

Figure 4.1: Main build tree. 


\subsubsection{Output build files}

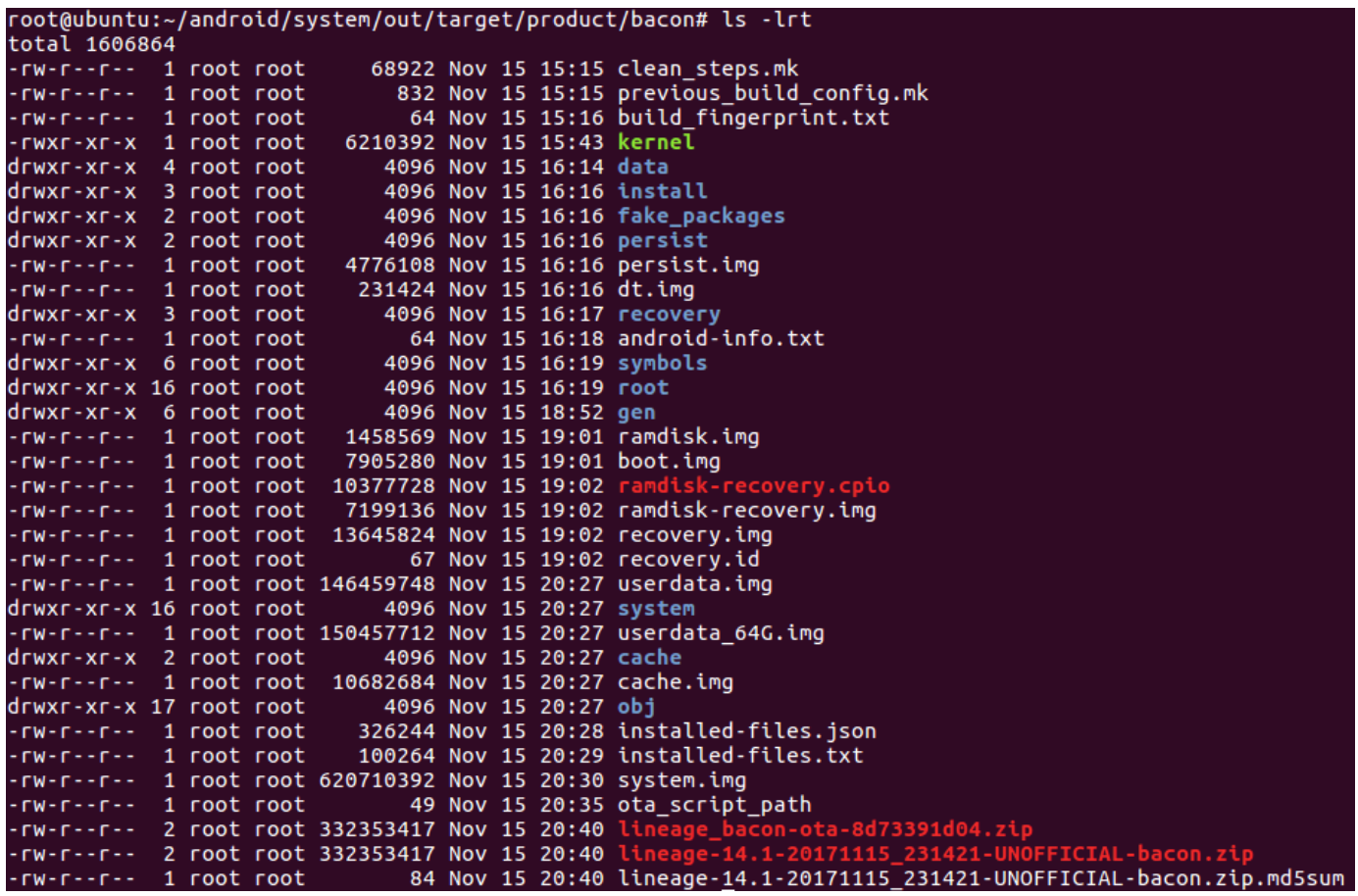

Figure 4.2: Final build files. 


\subsubsection{Extracting proprietary files}

Here I show the proprietary files shipping in the existing LineageOS pre-built image for the OnePlus One. All the files are categorized under different processes. Following are the details about the proprietary files.

- ADSP is the Application DSP (digital signal processor), which allows the device to process simple sets of data on low power, without having to wake up the Application processor (AP). It can run custom applications to perform simple tasks, such as word detection for voice recognition, sensor data aggregation from the accelerometer and gyroscope, and is also utilized for low power music playback. In the device it include different files are as follows:

- bin/adsprped

- vendor/lib/libadsprpc.so

- vendor/lib/libfastcvadspstub.so

- vendor/lib/libfastcvopt.so

- Bluetooth uses different libraries, as follows:

- bin/btnvtool

- bin/hciqcomminit

- vendor/lib/libbtnv.so

- Audio libraries include the following:

- vendor/lib/libacdbloader.so

- vendor/lib/libacdbrtac.so

- vendor/lib/libadiertac.so

- vendor/lib/libaudcal.so

- vendor/lib/soundfx/libqcbassboost.so

- vendor/lib/soundfx/libqcreverb.so

- vendor/lib/soundfx/libqcvirt.so

- DRM libraries are as follows:

- bin/qseecomd

- lib/libdrmdecrypt.so

- vendor/lib/libQSEEComAPI.so

- vendor/lib/libdrmfs.so

- vendor/lib/libdrmtime.so

- vendor/lib/librpmb.so

- vendor/lib/libssd.so

- vendor/lib/libwvdrmL3.so 
- vendor/lib/libwvm.so

- vendor/lib/libWVStreamControlAPIL3.so

- vendor/lib/drm/libdrmwvmplugin.so

- vendor/lib/mediadrm/libwvdrmengine.so

- Graphic libraries and firmwares files are as follows:

- etc/firmware/a330pfp.fw

- etc/firmware/a330pm4.fw

- vendor/lib/egl/eglsubAndroid.so

- vendor/lib/eg1/libEGLadreno.so

- vendor/lib/eg1/libGLESv1CMadreno.so

- vendor/lib/egl/libGLESv2adreno.so

- vendor/lib/egl/libq3dtoolsadreno.so

- vendor/lib/libadrenoutils.so

- vendor/lib/libbccQTI.so

- vendor/lib/libC2D2.so

- vendor/lib/libCB.so

- vendor/lib/libc2d30-a3xx.so

- vendor/lib/libgsl.so

- vendor/lib/libllvm-qcom.so

- vendor/lib/libOpenCL.so

- vendor/lib/libsc-a3xx.so

- vendor/lib/libRSDriveradreno.so

- vendor/lib/librsadreno.so

- vendor/lib/librsadrenosha1.so

- vendor/lib/libscale.so

- vendor/lib/libuiblur.so

- The listen service files are as follows:

- vendor/lib/liblistenhardware.so

- vendor/lib/liblistenjni.so

- vendor/lib/liblisten.so

- vendor/lib/liblistensoundmodel.so

- Media libraries on the device include files, are as follows:

- vendor/lib/libDivxDrm.so

- vendor/lib/libmm-color-convertor.so 
- vendor/lib/libmmosal.so

- vendor/lib/libSHIMDivxDrm.so

- Sensors libraries on the device include the following:

- bin/sensors.qcom

- vendor/lib/hw/activityrecognition.msm8974.so

- vendor/lib/hw/sensors.msm8974.so

- vendor/lib/libsensor1.so

- vendor/lib/libsensorreg.so

- Time services on the device include:

- bin/timedaemon

- vendor/lib/libtimegenoff.so

- vendor/lib/libTimeService.so

- app/TimeService/TimeService.apk

- WiFi firmwares files are as follows:

- lib/libwpaqmieapproxy.so

- lib/libwcnssqmi.so

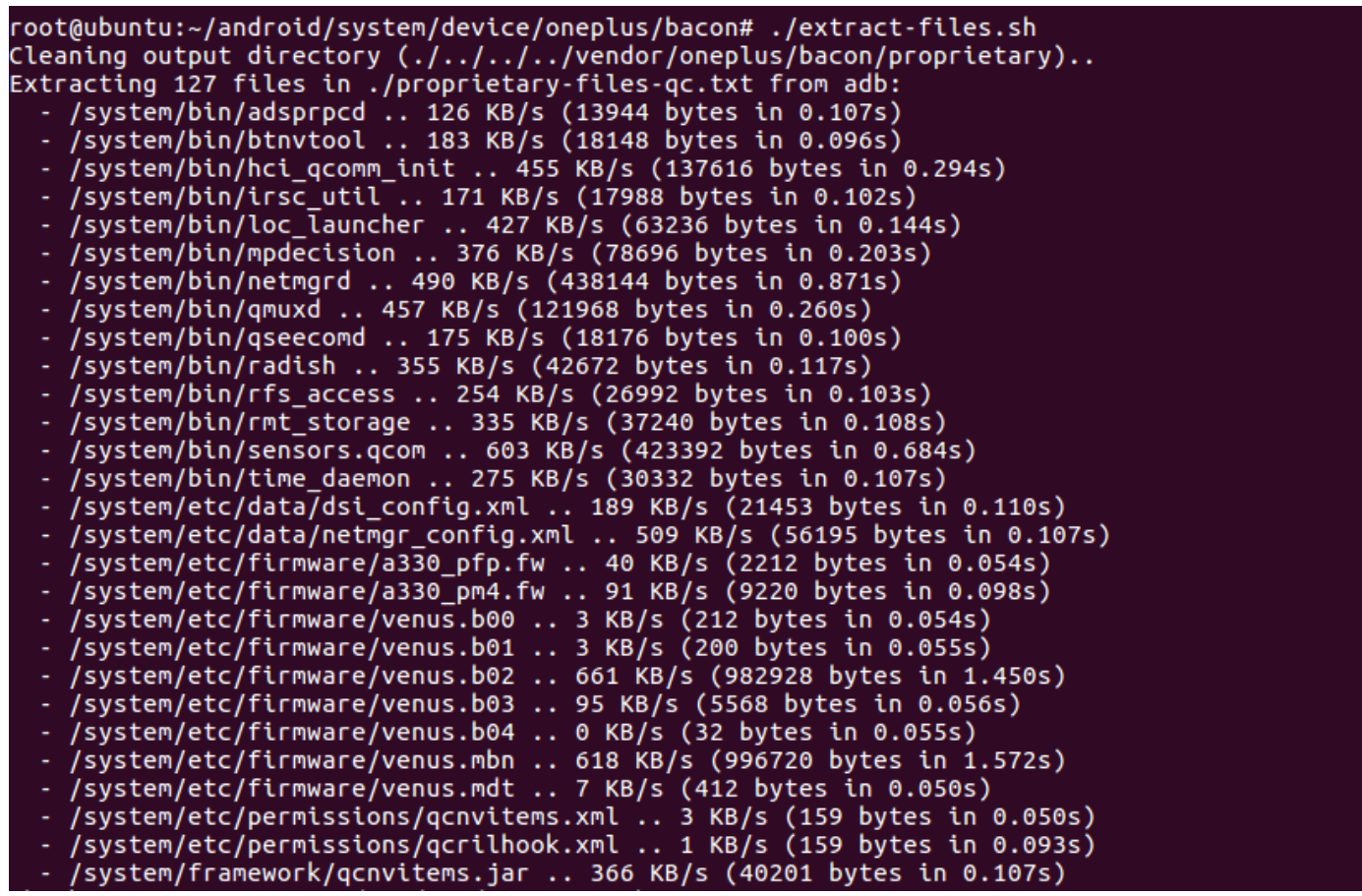

Figure 4.3: Proprietary files -1 . 


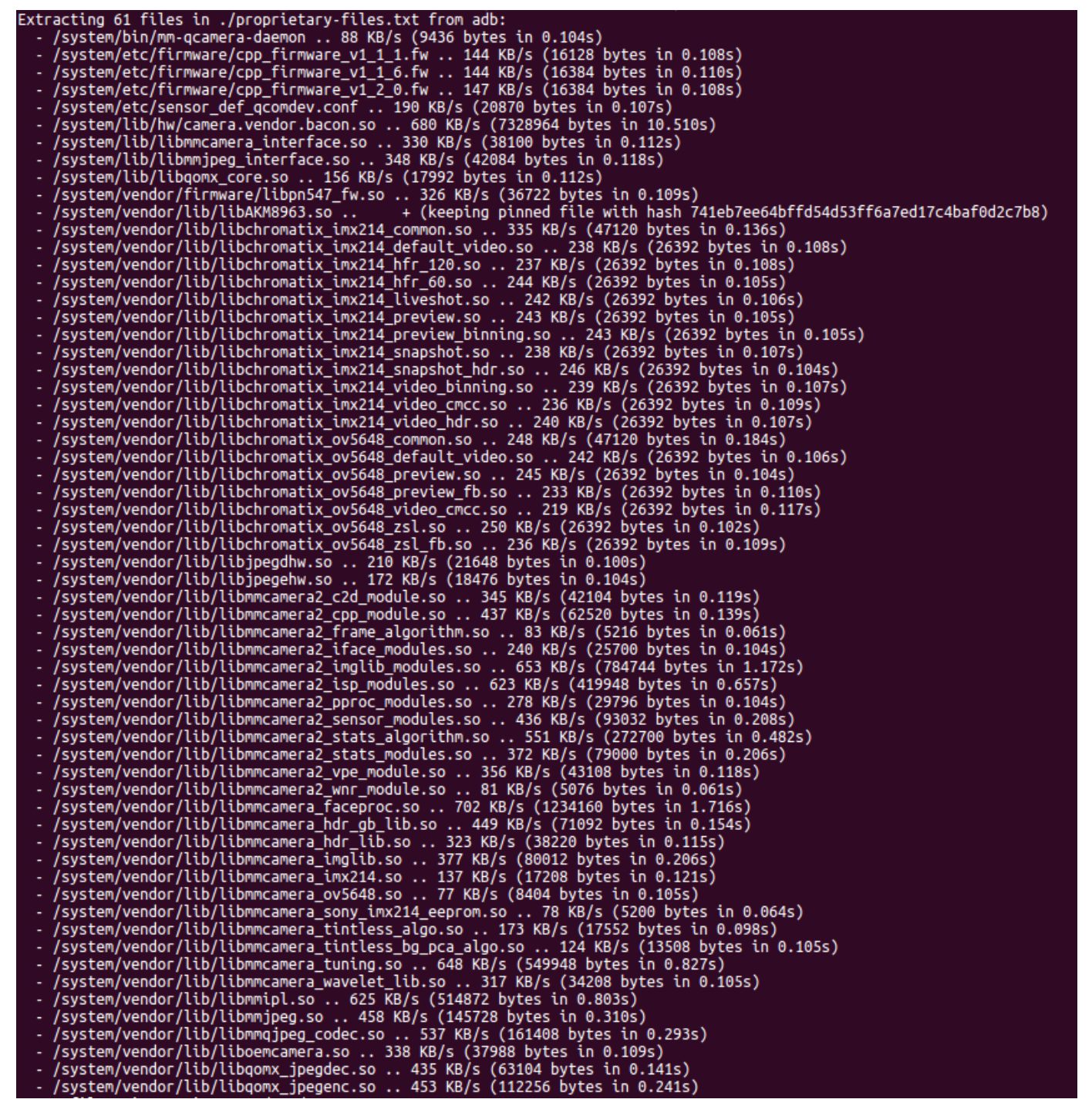

Figure 4.4: Proprietary files (listing 2). 
Table 4.1: OnePlus One mobile specifications.

\begin{tabular}{||l||l||}
\hline Parameter & Description \\
\hline \hline Codename & Bacon \\
\hline \hline Screen & 5.5 ' IPS-LCD \\
\hline \hline Resolution & $1080 x 1920$ \\
\hline \hline Soc & Qualcomm MSM8974AC \\
\hline \hline CPU & Snapdragon 801 \\
\hline \hline GPU & Quad-core 2.5 GHz Krait 400 \\
\hline \hline RAM & Adreno 330 \\
\hline \hline Storage & $3 \mathrm{~GB}$ \\
\hline \hline Rear Camera & $64 \mathrm{~GB}$ \\
\hline \hline Front Camera & $13 \mathrm{MP}$ \\
\hline \hline Battery & $5 \mathrm{MP}$ \\
\hline
\end{tabular}




\section{Chapter 5}

\section{Discussion and Conclusion}

\subsection{Discussion}

In this special study, I observe that the challenges for LineageOS are increasing compared to the stock Google Android variants. Although the alternative Google Play Services and client applications compared to the official Play services, primarily due to the user experience, which could be improved. I also observe that Google proprietary policy on different devices has led to security vulnerabilities and violation of users' privacy. By installing and contributing to the development of alternatives to the Google Play Services such as the microG project, we can support reverse engineering of certain Google services such as back-end location service, push notification and messaging service, and network-based location services utilizing the Mozilla location service for geolocation.

\subsection{Conclusion}

This special study explores opportunities for contributing to the development of LineageOS as a fully open mobile operating system. I show how to build LineageOS, and the same procedure should be applicable to many different devices. I describe alternatives to the Google Play services. From previous work, I conclude that data privacy and security vulnerabilities from the Play Store have violated users' privacy, that closed-source models are unable to solve those issues, making it important to invest in open source alternatives to improve user experience and make them a truly viable alternatives. 


\section{References}

Anderson, M. (2006). Global positioning tech inspires do-it-yourself mapping project. http://news.nationalgeographic.com/news/2006/10/061018-street-maps.html. (Online; accessed 18-October-2006)

Erturk, E. (2013). A case study in open source software security and privacy: Android adware. World Congress on Internet Security, 189-191.

Johnson, D. (2016). TOR Browser 6.0 is released. https://blog.torproject.org/. (Online; accessed 30-May-2016)

Khokhlov, I., \& Reznik, L. (2017). Data security evaluation for mobile android devices. Proceeding of 20th Conference of FRUCT Association, 154-160.

MacAskill, E. (2013). NSA files decoded: Edward Snowden's surveillance revelations explained. https://www.theguardian.com/world/interactive/2013/nov/01/snowden-nsa-filessurveillance-revelations-decoded. (Online; accessed 1-November-2013)

Mozilla. (2014). Release notes blog. https://www.mozilla.org/en-US/firefox/33.1/releasenotes/. ([Online; accessed 10-November-2014])

Nardi, T. (2012). F-Droid: The Android Market That Respects Your Rights. https://web.archive.org/web/20131203061021/http://www.thepowerbase.com/2012/08/fdroid-the-android-market-that-respects-your-rights/. (Online; accessed 27-August-2012)

Schwartz, B. (2014). Big win for duckduckgo: Apple adding to safari as private search option. https://searchengineland.com/big-win-duckduckgo-apple-adding-safari-private-searchoption-193081. (Online; accessed 2-June-2014)

Viennot, N., Garcia, E., \& Nieh, J. (2014). A measurement study of google play. Measurement and Modeling of Computer Systems - SIGMETRICS, 221-233. 